\title{
RESOLUÇÃO DO PROBLEMA DO CAIXEIRO VIAJANTE NO SETOR VAREJISTA USANDO HEURÍSTICA COM PREVISÃO DE TRAJETÓRIA
}

Traveling salesman problem resolution in the retail sector using heuristic with track forecast

Resolución de problemas de vendedores ambulantes en el sector minorista utilizando heurística con pronóstico de seguimiento
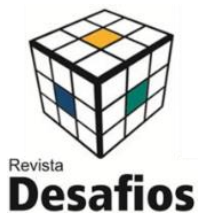

Artigo Original

Original Article

Artículo Original

\section{Wellington Gonçalves ${ }^{*}$, Hilson Henrique Massucatti Canal$^{2}$, Mauro Henrique Massucatti $\mathrm{Canal}^{3}$}

${ }^{1}$ Laboratório de Pesquisa Operacional, Logística e Transporte, Engenharia de Produção, Universidade Federal do Espírito Santo, São Mateus, Brasil.

*Correspondência: Laboratório de Pesquisa Operacional, Logística e Transporte, Universidade Federal do Espírito Santo, Rod. Gov. Mário Covas, Km 60, São Mateus, ES - Brasil. CEP 29932-540. e-mail

wellington.goncalves@ufes.br.

\section{RESUMO}

A entrega rápida e econômica em domicílio de mercadorias encomendadas é um desafio logístico. E, devido a isso, diversas empresas estão procurando novas formas de serem mais ágeis para com seus clientes. Em nossos cenários, a duração necessária para prestar serviço a qualquer cliente não é fixa, mas, definida como uma função do horário de início da atividade. Assim, este trabalho realizou uma simulação de rotas para reduzir distâncias percorridas em serviços de entrega, empregando a heurística Traveling Salesman Problem (TSP). O modelo proposto pode lidar com vários tipos de funções de tempo de serviço e, condicionantes e características locais. Além disso, aplicamos nosso modelo a várias instâncias reais com diferentes características e tamanhos de percurso. Os experimentos sugerem que são possíveis economias substanciais com esse modelo em comparação com a entrega baseada somente na expertise. A modelagem realizada e os resultados numéricos obtidos com a implementação do TSP em várias instâncias de teste são apresentados. Esses resultados permitiram identificar rotas otimizadas, as quais obtiveram uma redução média de $6,1 \%$ das distâncias percorridas, e ainda, possibilidades da aplicação desta metodologia como uma ferramenta de gestão e minimização de custos.

Palavras-chave: Mobilidade urbana. Otimização estocástica. Roteamento de veículo.

\section{ABSTRACT}

Fast and cost-effective home delivery of ordered goods is a logistical challenge. And because of this, many companies are looking for new ways to be more agile towards their customers. In our scenarios, the duration required to service any customer is not fixed, but is defined as a function of the activity's start time. Thus, this work performed a route simulation to reduce delivery service distances, using the Traveling Salesman Problem (TSP) heuristic. The proposed model can deal with various types of service time functions and local constraints and characteristics. In addition, we apply our model to many real instances with different characteristics and path sizes. Experiments suggest that substantial savings are possible with this model compared to delivery based only on expertise. The modeling performed and the numerical results obtained with the implementation of TSP in various test instances are presented. These results allowed the identification of optimized routes, which obtained an average reduction of $6.1 \%$ of the distances traveled, as well as possibilities of applying this methodology as a management and cost minimization tool. Keywords: Urban mobility. Stochastic optimization. Vehicle routing. 


\section{RESUMEN}

La entrega a domicilio rápida y rentable de los productos pedidos es un desafío logístico. Y debido a esto, muchas compañías están buscando nuevas formas de ser más ágiles con sus clientes. En nuestros escenarios, la duración requerida para atender a cualquier cliente no es fija, sino que se define en función de la hora de inicio de la actividad. Por lo tanto, este trabajo realizó una simulación de ruta para reducir las distancias del servicio de entrega, utilizando la heurística del Problema del Vendedor Ambulante (PVA). El modelo propuesto puede tratar varios tipos de funciones de tiempo de servicio y restricciones y características locales. Además, aplicamos nuestro modelo a muchas instancias reales con diferentes características y tamaños de ruta. Los experimentos sugieren que es posible un ahorro sustancial con este modelo en comparación con la entrega basada solo en la experiencia. Se presentan los modelos realizados y los resultados numéricos obtenidos con la implementación de PVA en varias instancias de prueba. Estos resultados permitieron la identificación de rutas optimizadas, que obtuvieron una reducción promedio del $6.1 \%$ de las distancias recorridas, así como las posibilidades de aplicar esta metodología como una herramienta de gestión y minimización de costos.

Descriptores: Movilidad urbana. Optimización estocástica. Enrutamiento de vehículos.

\section{INTRODUÇÃO}

Em sistemas de distribuição inseridos em ambientes urbanos, o fornecedor geralmente enfrenta diversas dificuldades em seu cotidiano, dentre elas, a incerteza relacionada à entrega de mercadorias e, a sua relação com a otimização de operação de seu depósito. A partir disso, de acordo com Sun et al. (2018), informações que proporcionem condições de elaborar alternativas de solução, desempenham um papel crucial em um planejamento de distribuição eficiente.

No Brasil, segundo o Serviço Brasileiro de Apoio às Micro e Pequenas Empresas (SEBRAE, 2018), a atividade de distribuição em meio urbano é indicada como um dos negócios promissores no país. O SEBRAE ainda destaca que, tal segmento possui elevado potencial de expansão a médio e, ao longo prazo, tendo por explicação o crescimento médio corresponde a 26\% ao ano no período de 2013 a 2017.

Assim, devido ao eminente crescimento da demanda por mercadorias e, em consequência disso, problemas relacionados a entrega, Bernardino e Paias (2018) citam que, devido a superpopulação urbana, é necessário que as organizações e empresas do setor varejista, desenvolvam e utilizem soluções que se adaptem a novas e diferentes realidades do mercado. Fato que, conforme sugerem Kaabi e Harrath (2019), deve ser tratado como um problema do roteamento de veículos, por estar diretamente ligado a situações e, também, a redes de distribuição. Estes autores ainda destacam que esse tipo de solução, além de atender demandas e necessidades de clientes, possibilitam vantagem competitiva frente a concorrência.

Para tanto, é necessário que se realize um planejamento adequado das redes de distribuição, assim como, das alternativas de unitização de entregas ponto a ponto, as quais promoverão subsídios para processos de tomada de decisão nas organizações (KINABLE et al., 2017). Corroborando com essa visão, Dickson e Tillé (2016), sugerem que em casos de entregas ponto a ponto que envolvem cargas unitizadas, é aconselhado utilizar o Traveling Salesman Problem (TSP) - Problema do Caixeiro Viajante (PCV) como forma de solução simplificada para tais cargas.

Considerando os contextos apresentados nesta seção, este trabalho teve por objetivo realizar simulação de rotas para reduzir distâncias percorridas em serviços de entrega, por meio da resolução heurística do TSP de Dantzig e Ramser (1959), implementada por intermédio de um Software de modelagem de otimização para programação linear (LINGO® - versão trial). Como unidade de pesquisa foi utilizada uma empresa do setor varejista que realiza 
entregas periódicas num município localizado na microrregião nordeste do Estado do Espírito Santo.

\section{MATERIAIS E MÉTODOS}

Para operacionalização e desenvolvimento da pesquisa foi utilizada como unidade de pesquisa, uma microempresa que atua no ramo de comercialização e distribuição de água mineral no município de São Mateus - Espírito Santo (Brasil). Assim, as investigações transcorreram no sentido de elucidar a operacionalização do setor de distribuição desta microempresa, que possui recursos próprios para realização de entregas.

Desta forma, a problemática sobre a otimização de rotas de entregas dessa microempresa do ramo de comercialização e distribuição de água mineral, foi caracterizada por meio de entrevistas (questões fechadas) e, reuniões junto à empresa que serviu de unidade de pesquisa. A partir disso, foram selecionadas aleatoriamente quatro entregas em diferentes turnos, as quais foram realizadas entre os dias 13 e 15 de junho de 2019, em horário comercial. Para caracterizar as rotas utilizadas nestas entregas, foram obtidas as seguintes informações: horário de partida e de chegada do entregador na empresa; pontos de entrega e, caminhos utilizados pelo entregador.

De tal modo, a obtenção de dados para resolução da otimização de rotas de entregas, se deu a partir das distâncias percorridas, além das distâncias entre todos os pontos atendidos. Na sequência, houve a seleção do caminho mais adequado para execução dessas rotas, considerando funcionalidades (movimentação e armazenamento) e princípios fundamentais do transporte (economia de distância e economia de escala). Para obtenção de tais rotas foi empregada a ferramenta de roteirização do Google Maps (GOOGLE, 2019). E, posteriormente, para representam as distâncias entre todos os pontos de cada rota, foi elaborada uma matriz Origem-Destino (OD) do tipo "De-Para".

Por fim, após obtenção da matriz OD, o problema do Caixeiro Viajante foi modelado no LINGO® Software de modelagem de otimização para programação linear, não linear e inteira - versão trial, a fim de auxiliar na operacionalização da resolução deste. As abordagens de modelagens e, implementação da heurística TSP foram executadas em um Intel Core I5 2.70 GHz com $16 \mathrm{~GB}$ de RAM, equipado com quatro núcleos. Para permitir comparações justas futuras, todos os experimentos foram realizados definindo como um dado número de experimentos.

\section{RESULTADOS E DISCUSSÃO}

A empresa de comercialização e distribuição de água mineral que foi utilizada como unidade de pesquisa está situada no bairro Guriri (São Mateus Espírito Santo /Brasil). Devido à confiabilidade das condições de oferta de água potável não serem adequadas, há cada vez mais um aumento na demanda por água mineral nesse bairro.

Assim, após compilação de informações das reuniões com os gestores da empresa (serviu de unidade de pesquisa), foi possível mapear 4 (quatro) rotas. Sendo estas foram apontadas como principais gargalos em termos de movimentação e operação. Desta forma, tais rotas foram caracterizadas considerando a empresa como ponto de partida e chegada (Tabela 1). 
Tabela 1. Mapeamento da programação de entregas.

\begin{tabular}{|c|c|c|c|c|}
\hline Rota & $\begin{array}{l}\text { Horário de } \\
\text { partida* }\end{array}$ & $\begin{array}{l}\text { Horário de } \\
\text { chegada do } \\
\text { entregador* }\end{array}$ & $\begin{array}{l}\text { Distância entre pontos de } \\
\text { entrega (metros) }\end{array}$ & Caminhos utilizados** \\
\hline 1 & $10 \mathrm{~h} 40$ & $11 \mathrm{~h} 13$ & $\begin{array}{l}\text { A:B (1000); B:C (1300); C:D } \\
\text { (1200); D:E (700) e E:A (700). }\end{array}$ & $\begin{array}{l}(-18.740082,-39.751171) ;(-18.742900, \\
-39.754904) ;(-18.736609,-39.758134) ; \\
(-18.735914,-39.748620) ;(-18.737822, \\
-39.753501)\end{array}$ \\
\hline 2 & $16 \mathrm{~h} 30$ & $16 \mathrm{~h} 55$ & $\begin{array}{l}\text { A:B (600); B:C (1200); C:D } \\
(900) \text { e D:A (1000). }\end{array}$ & $\begin{array}{l}(-18.740082,-39.751171) ;(-18.736279 \\
-39.751006) ;(-18.734936,-39.755130) \\
(-18.742443,-39.754887)\end{array}$ \\
\hline 3 & 9h15 & $9 \mathrm{~h} 46$ & $\begin{array}{l}\text { A:B (1000); B:C (850); C:D } \\
(850) ; \mathrm{D}: \mathrm{E}(500) \text { e E:A (450). }\end{array}$ & $\begin{array}{l}(-18.740082,-39.751171) ;(-18.743230 \\
-39.747721) ;(-18.743903,-39.754320) \\
(-18.736262,-39.753947) ;(-18.737345 \\
-39.750418)\end{array}$ \\
\hline 4 & $14 \mathrm{~h} 32$ & $15 \mathrm{~h} 03$ & $\begin{array}{l}\text { A:B (1300); B:C (700); C:D } \\
(650) ; \mathrm{D}: \mathrm{E}(550) \text { e E:A (750). }\end{array}$ & $\begin{array}{l}(-18.740082,-39.751171) ;(-18.745337, \\
-39.751356) ;(-18.743950,-39.755426) ; \\
(-18.743498,-39.750036) ;(-18.741313, \\
-39.753238) .\end{array}$ \\
\hline
\end{tabular}

(*) Em relação a empresa. (**) Coordenadas geográficas.

A obtenção de dados para resolução da otimização de rotas de entregas, se deu a partir das distâncias percorridas, além das distâncias entre todos pontos atendidos. A partir disso, foi realizada escolha dos caminhos a serem percorridos para realização dessas rotas, tendo por princípio a realização de movimentação e armazenamento de produtos, além de economia de distância e economia de escala. Dentro desse contexto, a Figura 2 apresenta a disposição dos pontos de entrega que foram atendidos pelo entregador da empresa, entre os dias 13 e 15 de junho de 2019, em horário comercial, os quais foram obtidos a partir da ferramenta de roteirização do Google Maps (GOOGLE, 2019).

Figura 2. Representação das rotas operacionalizadas pela empresa.
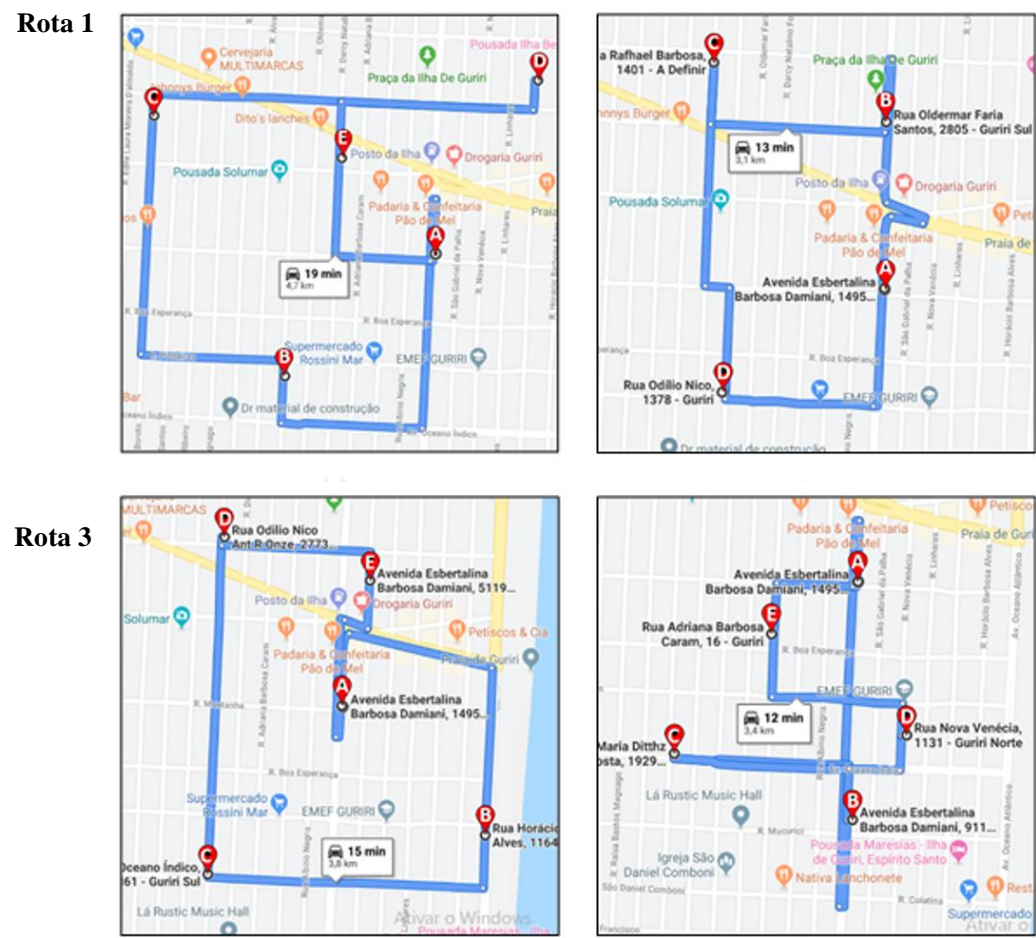
Assim, para todos os grafos (Figura 2), foram marcados pontos que representam as localizações das entregas, no qual a empresa é representada pelo ponto A. E, consequentemente, as ordens de entregas estão representadas pelos demais pontos em ordem alfabética crescente, ou seja, o ponto B representa a primeira entrega, $\mathrm{C}$ a segunda e, assim por diante. Posteriormente, avaliando as distâncias obtidas ponto a ponto, foi possível determinar as distâncias totais percorridas em cada rota (Rota 1: 4900m; Rota 2: 3700m; Rota 3: 3650m; e Rota 4: 3950m). Em seguida, foi desenvolvida uma matriz Origem-Destino (OD) do tipo "De-Para" para cada Rota, contendo as distâncias entre pontos (Tabelas 2 a 5).

Tabela 2. Compilação entre pontos - Rota 1 (metros).

\begin{tabular}{cccccc}
\hline Pontos & A & B & C & D & E \\
\hline A & 0 & 1000 & 1500 & 1200 & 700 \\
B & 1000 & 0 & 1300 & 1300 & 650 \\
C & 1500 & 1300 & 0 & 1200 & 650 \\
D & 1200 & 1300 & 1200 & 0 & 700 \\
E & 700 & 650 & 650 & 700 & 0 \\
\hline
\end{tabular}

Tabela 3. Compilação entre pontos - Rota 2 (metros).

\begin{tabular}{ccccc}
\hline Pontos & A & B & C & D \\
\hline A & 0 & 600 & 1100 & 1000 \\
B & 600 & 0 & 1200 & 1100 \\
C & 1100 & 1200 & 0 & 900 \\
D & 1000 & 1100 & 1200 & 0 \\
\hline
\end{tabular}

Tabela 4. Compilação entre pontos - Rota 3 (metros).

\begin{tabular}{cccccc}
\hline Pontos & A & B & C & D & E \\
\hline A & 0 & 1000 & 1100 & 900 & 450 \\
B & 1000 & 0 & 850 & 1600 & 1200 \\
C & 1100 & 850 & 0 & 850 & 1300 \\
D & 900 & 1600 & 850 & 0 & 500 \\
E & 450 & 1200 & 1300 & 500 & 0 \\
\hline
\end{tabular}

Tabela 5. Compilação entre pontos - Rota 4 (metros).

\begin{tabular}{cccccc}
\hline Pontos & A & B & C & D & E \\
\hline A & 0 & 1300 & 1300 & 700 & 750 \\
B & 1300 & 0 & 700 & 400 & 800 \\
C & 1300 & 700 & 0 & 650 & 600 \\
D & 700 & 400 & 650 & 0 & 550 \\
E & 750 & 800 & 600 & 550 & 0 \\
\hline
\end{tabular}

Assim, dando continuidade a realização da simulação de rotas, a resolução do Traveling Salesman Problem proposta por Dantzig e Ramser (1959) foi modelada no LINGO® (Figura 3). Essa modelagem permitiu analisar as condições atuais das rotas de entregas e, também, simular cenários.

Figura 3. Parametrização em modelagem do TSP.

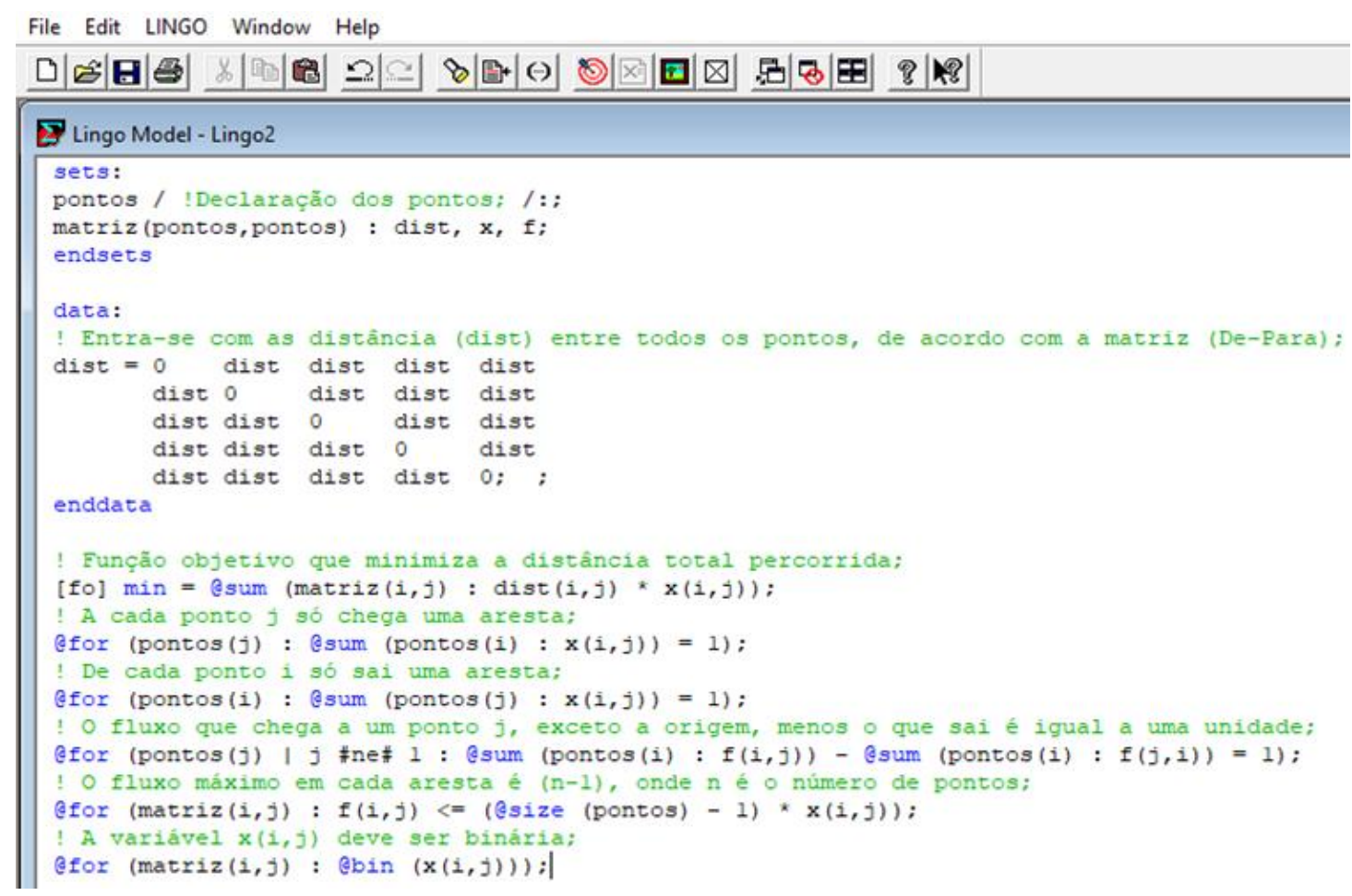


Ao serem simulados os dados das distâncias entre todos os pontos das Rotas (Tabelas 2 a 5), foram obtidas sugestões de solução ótima do TSP, as quais foram agrupadas em uma matriz binária, em que a variável assume valor 1 , se a aresta (caminho) for selecionada na solução e, em caso contrário a variável assume valor 0. Dessa maneira, por meio da modelagem construída no LINGO®, as soluções otimizadas de menor distância para cada Rota foram obtidas. Para a Rota 1, após 34 iterações realizadas em menos de um segundo, o roteiro de destinos foi o seguinte: Ponto A - Ponto D - Ponto C - Ponto E Ponto B - Ponto A (Tabela 6), o qual resultou numa distância total de 4700 metros.

Tabela 6. Matriz binária e solução otimizada da Rota 1.

\begin{tabular}{cccccc}
\hline Pontos & $\mathbf{A}$ & $\mathbf{B}$ & $\mathbf{C}$ & $\mathbf{D}$ & $\mathbf{E}$ \\
\hline A & 0 & 1 & 0 & 0 & 0 \\
B & 0 & 0 & 0 & 0 & 1 \\
C & 0 & 0 & 0 & 1 & 0 \\
D & 1 & 0 & 0 & 0 & 0 \\
E & 0 & 0 & 1 & 0 & 0
\end{tabular}

Ao simular os dados relativos à Rota 2 , a distância total obtida foi a mesma da executada inicialmente pelo entregador, ou seja, 3700 metros percorridos. Esse resultado foi percebido após 22 iterações, as quais foram realizadas em menos de um

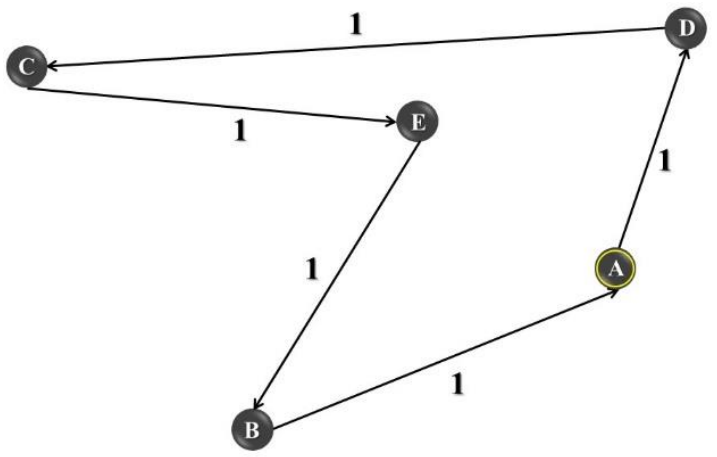

segundo, que resultou na rota de menor custo (distância). Sugerindo que o atual percurso possa ser o ideal a ser utilizado, entretanto, o LINGO® indicou a seguinte sequência (Tabela 7): Ponto A - Ponto D Ponto C - Ponto B - Ponto A.

Tabela 7. Matriz binária e solucão da Rota 2

\begin{tabular}{ccccc}
\hline Pontos & A & B & C & D \\
\hline A & 0 & 1 & 0 & 0 \\
B & 0 & 0 & 1 & 0 \\
C & 0 & 0 & 0 & 1 \\
D & 1 & 0 & 0 & 0
\end{tabular}

Por meio da compilação da modelagem do TSP no LINGO® e, após 89 iterações, realizadas em menos de um segundo, obteve-se a solução otimizada para o problema, resultando na Rota 3 de menor custo (distância). Ao serem verificados os resultados se identificou que a distância total é análoga à executada

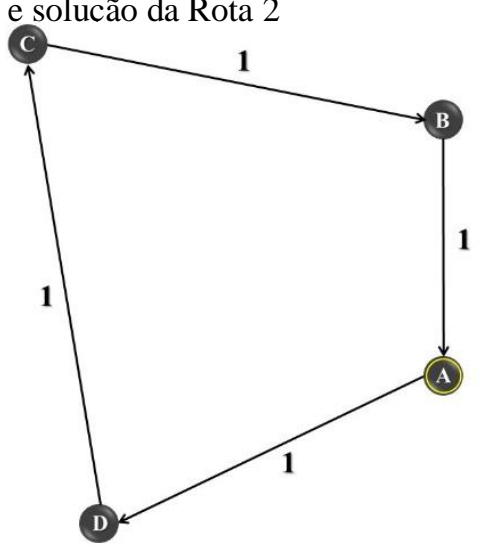

inicialmente, ou seja, 3650 metros. Havendo ainda a sugestão da seguinte sequência de atendimento (Tabela 8): Ponto A - Ponto E - Ponto D - Ponto C - Ponto B - Ponto A. 
Tabela 8. Matriz binária e solução da Rota 3.

\begin{tabular}{cccccc}
\hline Pontos & $\mathbf{A}$ & $\mathbf{B}$ & $\mathbf{C}$ & $\mathbf{D}$ & $\mathbf{E}$ \\
\hline A & 0 & 1 & 0 & 0 & 0 \\
B & 0 & 0 & 1 & 0 & 0 \\
C & 0 & 0 & 0 & 1 & 0 \\
D & 0 & 0 & 0 & 0 & 1 \\
E & 1 & 0 & 0 & 0 & 0
\end{tabular}

Por fim, a simulação da Rota 4, após 92 iterações realizadas em um tempo de um segundo, resultou numa distância total de 3150 metros, com o

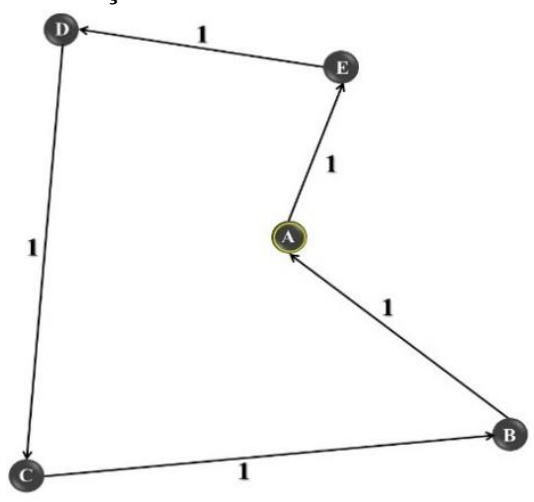

seguinte atendimento sequencial (Tabela 9): Ponto A Ponto E - Ponto C - Ponto B - Ponto D - Ponto A.

Tabela 9. Matriz binária e solução da Rota 4.

\begin{tabular}{cccccc}
\hline Pontos & A & B & $\mathbf{C}$ & $\mathbf{D}$ & $\mathbf{E}$ \\
\hline A & 0 & 0 & 0 & 1 & 0 \\
B & 0 & 0 & 1 & 0 & 0 \\
C & 0 & 0 & 0 & 0 & 1 \\
D & 0 & 1 & 0 & 0 & 0 \\
E & 1 & 0 & 0 & 0 & 0
\end{tabular}

Para as quatro Rotas utilizadas neste trabalho foi possível alcançar uma redução média de $6,1 \%$ da atual distância percorrida. Entretanto, pode-se notar que para a Rota 4 obteve uma redução mais elevada, na ordem de 20,3\%. Seguida da Rota 1 que teve uma redução de 4,1\%, sendo que nas Rotas 2 e 3 não houveram incrementos ou redução de distâncias. Suscitando que, estes resultados contribuem para melhoras de processos de operacionalização de movimentação e armazenamento, além de economias de distância e, de escala, fato que influencia diretamente o transporte e custos da empresa.

\section{CONCLUSÃO}

A alocação estratégica de atendimentos numa programação de entregas para clientes é um problema importante que surge no contexto das operações de roteamento de veículos. E, devido a variações da DOI: http://dx.doi.org/10.20873/uftv7-7804 demanda, associadas a outras dimensões como polos geradores de demanda influenciam e impactam significativamente as operações diárias das empresas. Com isso, o distribuidor deve se esforçar para entender o seu contexto e, propor soluções que se adequem a sua realidade, da melhor maneira possível, uma vez que não fazer isso pode resultar em significativas repercussões na sua reputação.

Nesse sentido, é importante reconhecer e incorporar no planejamento de operações de entregas, a incerteza inerente em parâmetros como demandas do cliente, condições de vias e tempos de viagem, cujas caraterísticas e comportamentos reais somente são conhecidos no dia e momento da operação. Dentro desse contexto, este trabalho traz uma significativa contribuição de baixo custo a empresas do setor e, outras que atuam com entregas nas mesmas proporções. E, esta contribuição, também pode ser Revista Desafios - v. 7, n. 3, 2020 
parametrizada e, adequada a distintas aplicações e variações, como janelas de tempo, dentre outras.

Por isso, o objetivo deste trabalho pode ser considerado como atingido, não somente por ter efetivado de simulação de rotas, como também, ter minimizado distâncias percorridas e, consequentemente, proporcionar aos gestores da empresa unidade de pesquisa, condições de otimização nas abordagens operacionais, técnicas e financeiras. Isso foi possível ao definimos um modelo de programação linear inteiro e, explicitarmos condicionantes locais parametrizadas para o Traveling Salesman Problem (TSP) e o problema de roteamento de veículos.

Desenvolvemos uma abordagem heurística de ramificação e aplicação do TSP. Outros experimentos computacionais existentes na literatura em instâncias de tamanho realista, mostraram que a obtenção da solução exata dessa variante de problema é extremamente desafiadora, devido aos stakeholders, dimensões e variáveis que compõe o arcabouço de estudo. No entanto, por meio dos experimentos realizados, vimos que soluções aproximadas de boa qualidade (e, portanto, particularmente úteis para profissionais do setor, empresas que atuam com entregas e academia) podem ser obtidas em curtos tempos de processamento, com custos de operacionalização de soluções adequados à diferentes realidades. Desenvolvimentos futuros podem estender as investigações ao caso de vários veículos.

\section{AGRADECIMENTO}

Agradecemos ao Laboratório de Pesquisa Operacional, Logística e Transporte (POLT) da Universidade Federal do Espírito Santo (UFES) / Centro Universitário Norte do Espírito Santo (CEUNES) pelo apoio acadêmico e técnico na concepção e desenvolvimento deste trabalho. E, também, a Fundação de Amparo À Pesquisa e
Inovação do Espirito Santo por meio dos Editais FAPES-CNPq 13/2018 - PICJr e FAPES 21/2018 Universal.

Todos os autores declararam não haver qualquer potencial conflito de interesses referente a este artigo.

\section{REFERÊNCIAS}

BERNARDINO, R. e PAIAS, A. Solving the family traveling salesman problem. Eur. Jour. of Oper. Res., v.267, n.2, p.453-466, 2018.

BRAEKERS, K.; RAMAEKERS, K. e VAN NIEUWENHUYSE, I. The vehicle routing problem: State of the art classification and review. Comp. Ind. Eng., v.99, p.300-313, 2016.

BRASIL. Lei $\mathrm{n}^{\mathrm{o}}$ 12.587, de 3 de janeiro de 2012 . Institui as diretrizes da Política Nacional de Mobilidade Urbana. Diário Oficial [da] República Federativa do Brasil: seção 1, Brasília, DF, seção 1, p. 1,04 jan. 2012.

ÇOLAK, S.; LIMA, A. e GONZÁLEZ, M.C. Understanding congested travel in urban areas. Nat. com., v. 7, p. 1-8, 2016.

DANTZIG, G.B. e RAMSER, J.H. The truck dispatching problem. Manag. Sci., v. 6, n. 1, p. 80-91, 1959.

DORIGO, M. e GAMBARDELLA, L.M. Ant colony system: a cooperative learning approach to the traveling salesman problem. IEEE Trans. evol. comp., v. 1, n. 1, p. 53-66, 1997.

KINABLE, J.; SMEULDERS, B.; DELCOUR, E. e SPIEKSMA, F.C. Exact algorithms for the equitable traveling salesman problem. Eur. Jour. Op. Res., v. 261, n. 2, p. 475-485, 2017.

DICKSON, M.M. e TILLÉ, Y. Ordered spatial sampling by means of the traveling salesman problem. Comput. Stat., v. 31, n. 4, p. 1359-1372, 2016.

ELGESEM, A.S.; SKOGEN, E.S.; WANG, X. e FAGERHOLT, K. A traveling salesman problem with pickups and deliveries and stochastic travel times: An application from chemical shipping. Eur. Jour. Op. Res., v. 269, n. 3, p. 844-859, 2018.

GOOGLE, INC. Google Maps. 2019. Disponível em: $<$ http://code.google.com/apis/maps/documentation/di rections/>. Acesso: 14 jun. 2019. 
HAVENGA, J.H.; BOD, A. e SIMPSON, Z.P.A logistics barometer for South Africa: towards sustainable freight mobility. Jour. Transp. Supp. Chain Mana., v. 10, n. 1, p. 1-7, 2016.

IBGE - Instituto Brasileiro de Geografia e Estatística. Censo Demográfico 2010. Disponível em: $<$ https://censo2010.ibge.gov.br/>. Acesso: 14 jun. 2019.

KAABI, J. e HARRATH, Y. Permutation rules and genetic algorithm to solve the traveling salesman problem. Arab. Jour. Basic. Appl. Sci., v. 26, n. 1, p. 283-291, 2019.

KAMARGIANNI, M.; LI, W.; MATYAS, M. e SCHÄFER, A. A critical review of new mobility services for urban transport. Transp. Res. Proc., v. 14, p. 3294-3303, 2016.

LUEDERITZ, C.; SCHAPKE, N.; WIEK, A.; LANG, D.J.; BERGMANN, M.; BOS, J.J.; BURCH， S.; DAVIES, A.; ... e WESTLEY, F.R. Learning through evaluation-A tentative evaluative scheme for sustainability transition experiments. Jour. Clea. Prod., v. 169, p. 61-76, 2017.

MÄKINEN, K.; KIVIMAA, P. e HELMINEN, V. Path creation for urban mobility transitions: Linking aspects of urban form to transport policy analysis. Man. Environ. Qual. An Intern. Jour., v. 26, n. 4, p. 485-504, 2015.

MARTORELLI, M.; COSTA, A.G.V. e SÁ, A.C.B. A inclusão do transporte de cargas no sistema nacional de mobilidade urbana. Revis. LOGS: Log. Oper. Glob. Sust., v. 1, n. 1, p. 182-197, 2019.

MCCORMICK, K.; ANDERBERG, S.; COENEN, L. e NEIJ, L. Advancing sustainable urban transformation. Jour. Clea. Prod., v. 50, p. 1-11, 2013.

MURRAY, C.C. e CHU, A.G. The flying sidekick traveling salesman problem: Optimization of droneassisted parcel delivery. Transp. Res. Part C: Emer. Techn., v. 54, p. 86-109, 2015.

OLIVEIRA, L.K.; SOUZA HENRIQUES, R.; OLIVEIRA, R.S. e DENAIS, M. Análise dos benefícios de um espaço logístico urbano na distribuição urbana de mercadorias. Rev. Prod. Online, v. 16, n. 3, p. 988-1006, 2016.

RODRIGUE, J.P.; COMTOIS, C. e SLACK, B. The geography of transport systems. Routledge, 2016.

SAWIK, B.; FAULIN, J.; PÉREZ-BERNABEU, E. A multicriteria analysis for the green VRP: A case discussion for the distribution problem of a Spanish retailer. Transp. Res. Proc., v. 22, p. 305-313, 2017.

SEBRAE - Serviço Brasileiro de Apoio às Micro e Pequenas Empresas. Conheça os negócios mais promissores para 2018. Disponível em: $<$ https://www.sebrae.com.br $>$. Acesso em: 05 jul. 2019.

SILVA, D.M.; MELLO BANDEIRA, R.A. e CAMPOS, V.B.G. Avaliando a sustentabilidade alcançada por meio de entregas noturnas: o caso da distribuição de combustíveis na cidade do Rio de Janeiro. TRANSP., v. 26, n. 2, p. 54-69, 2018.

SINAY, M.C.F.; CAMPOS, V.B.G.; DEXHEIMER, L. e NOVAES, A.G. Distribuição de carga urbana: componentes, restrições e tendências. Conselho Nacional de Desenvolvimento Científico e Tecnológico, p. 1-9, 2004.

SOUSA, M.C.C. e PENNISI FILHO, R.R. A importância do planejamento para a gestão pública do espaço urbano sustentável. Idea, v. 8, n. 1, p. 1-11, 2017.

STIGLIC, M.; AGATZ, N.; SAVELSBERGH, M. e GRADISAR, M. Enhancing urban mobility: Integrating ride-sharing and public transit. Comp. Oper. Res., v. 90, p. 12-21, 2018.

SUN, L.; KARWAN, M.H. e DIABY, M. The indefinite period traveling salesman problem. Eur. Jour. Oper. Res., v. 270, n. 3, p. 1171-1181, 2018.

TAILLARD, É.D. e HELSGAUN, K. POPMUSIC for the travelling salesman problem. Eur. Jour. Oper. Res., v. 272, n. 2, p. 420-429, 2019.

VEENSTRA, M.; ROODBERGEN, K.J.; VIS, I.F. e COELHO, L.C. The pickup and delivery traveling salesman problem with handling costs. Eur. Jour. Oper. Res., v. 257, n. 1, p. 118-132, 2017. 